\title{
Incidence of Metachronous Distant Metastasis and ypN Classification Influence Patient Survival in Endosonographically Confirmed uT3 Rectal Cancer after Neoadjuvant Therapy and Ro Resection: A Historical Cohort Analysis
}

\author{
Achim Troja $^{a}$ Hans-Günther Hempen ${ }^{b} \quad$ Mareike Demmer $^{c} \quad$ Dalibor Antolovic $^{a}$ \\ Hans-Rudolf Raaba \\ a University Department of General and Visceral Surgery, Clinical Centre of Oldenburg, Oldenburg, Germany; \\ ${ }^{b}$ Department of General and Visceral Surgery, St. Josefs Hospital Cloppenburg, Cloppenburg, Germany; \\ ${ }^{c}$ Department of Urology, Clinical Centre of Oldenburg, Oldenburg, Germany
}

\section{Keywords}

Multimodal therapy $\cdot$ Response rate $\cdot$ Total mesorectal excision - Tumor regression

\section{Summary}

Background: Tumor response after neoadjuvant radiochemotherapy (NRC) prior to surgery and other parameters are likely to have an influence on the survival rate of patients suffering from T3 rectal cancer. Methods: 51 patients (17 female, 34 male; 59.0 years; Apache < 9 points: 95.1\%; ASA I-II 88.3\% and ASA III 11.8\%) were treated with NRC (50.4 Gy; 5-fluorouracil/folinic acid) 4-6 weeks prior to surgery because of UT3 rectal cancer (G2: 96\%; adenocarcinoma $86.3 \%$; cUICC I| $62.7 \%$ ). NRC led to a tumor response (TR) (ypT0-ypT2) in 45.1\% (ypTONOM0 $7.8 \%)$. Results: Neither the age of patients nor Apache/ ASA score, histology, UICC staging, ypTNM, Dukes staging, infiltration of vessels, surgical procedure, local recurrence nor TR had a significant influence on the patients' survival time. Patients with metachronous distant metastasis (MDM) during the follow-up period (mean: 8.2 years; 1 month to 14.5 years) and patients with ypN1ypN2 had a significantly shorter survival time. Conclusions: NRC prior to surgery leads to a remarkable TR rate but has no significant impact of TR on the patients' survival time. Occurrence of MDM during the follow-up pe-

Achim Troja and Hans-Günther Hempen are joint first authors of this paper. riod and ypN1/N2 status do have a greater influence. It is necessary to investigate larger cohorts of patients in the future to obtain more conclusive results and to define factors with influence on survival.

(c) 2016 S. Karger GmbH, Freiburg

\section{Introduction}

In Germany, rectal cancer represents 15.4 and $12.6 \%$ of all tumors in females and males, respectively. Each year more than 27,000 men and 30,000 women are newly diagnosed with colorectal cancer. The incidence of 70 cases per 100,000 inhabitants for this disease remained nearly unchanged during the last 20 years. The mortality rate is about $37 / 100,000$ persons. The overall 5 -year survival rate of rectal cancer is $45 \%$ (males) and $47 \%$ (females) $[1,2]$.

According to Kerr et al. [3], the UICC classification is the most important prognostic factor for patient survival. The 5-year relative survival rate in UICC I tumors is $96.9 \%$. It decreases to $75.5 \%$ (UICC II), 58\% (UICC III), and 6\% (UICC IV) if the UICC staging is increasing.

Several treatment options are recommended for rectal carcinomas [4]. Local excision is likely to be curative in patients with a primary rectal tumor staged T1N0M0, without signs of high-risk features (i.e. poorly differentiated, vascular and/or neural invasion). If the tumor invades the muscularis propria (T2N0M0), radical rectal resection is recommended. Patients with transmural and/or node-positive rectal cancer (T3/T4 with or without N1) and 
Table 1. Demographic and clinical patient characteristics at baseline

\begin{tabular}{lc}
\hline Variable & $\begin{array}{l}\text { Mean } \pm \text { SEM (median; range) } \\
\text { or number }(\%)\end{array}$ \\
\hline $\begin{array}{l}\text { Age at diagnosis, years } \\
\text { Age at surgery, years }\end{array}$ & $58.7 \pm 1.2(58.0 ; 25.7-73.3)$ \\
Gender & $59.0 \pm 1.3(58.3 ; 25.9-73.6)$ \\
$\quad$ Female & $17(33.3 \%)$ \\
$\quad$ Male & $34(66.7 \%)$ \\
Apache score, points & $30(73.2 \%)$ \\
$0-4$ & $9(21.9 \%)$ \\
5-9 & $2(4.9 \%)$ \\
10 & $6(11.8 \%)$ \\
ASA score & $39(76.4 \%)$ \\
ASA I & $6(11.8 \%)$ \\
ASA II & \\
ASA III & \\
\hline SEM $=$ Standard error of the mean. &
\end{tabular}

presenting no distant metastases should be treated by means of preoperative chemoradiation followed by radical resection [5].

This retrospective study investigates patient survival rates after neoadjuvant radiochemotherapy (NRC) applied prior to R0 resection. This consists of a conventionally fractioned radiotherapy (up to $50 \mathrm{~Gy}$ ) and additional chemotherapy (5-fluorouracil (FU) and folinic acid) followed by surgery within 4-6 weeks, and is analog to the so-called CAO/ARO/AIO-94 scheme reported by Sauer [6].

Herein, we report the influence of age, histologic findings, tumor staging, grading, tumor response, local recurrence, and distant metastasis on the survival of patients.

\section{Patients and Methods}

\section{Patients}

We enrolled patients treated from February 1991 to December 1997 for rectal cancer in the Department of General and Visceral Surgery, Clinical Center of Oldenburg, Germany, in this retrospective study.

\section{Including Criteria}

Endosonographically confirmed T3 rectal cancer, curative elective surgery, R0 resection, metastatic-free disease prior to surgery.

\section{Treatment}

All patients underwent an NRC (up to $50.4 \mathrm{~Gy}$ ) and had an adjuvant chemotherapy consisting of 5-FU and folinic acid. 4-6 weeks after radiotherapy all patients were treated with a radical anterior rectal resection of less than $11 \mathrm{~cm}$ ( $\mathrm{n}=25 ; 49.0 \%$ ), an abdominoperineal rectal extirpation (APRE) ( $\mathrm{n}=21$; $41.2 \%)$, a pull-through procedure $(\mathrm{n}=3 ; 5.9 \%)$, or a laparoscopically assisted APRE $(n=2 ; 3.9 \%)$. All patients underwent the surgical procedures between 1991 and 1997, while the follow-up period was carried out between 1992 and 2005. Total mesorectal excision was performed in all cases.

\section{Tumor Response}

All patients had an endosonographically confirmed $\mathrm{T} 3$ rectal cancer before the beginning of NRC. Patients with a T0, T1, or T2 staging at the pathohistological investigation (postoperative) were defined as responders (ypT0-ypT2). Patients who had a ypT3 tumor were defined as non-responders.
Table 2. Tumor-related variables of patients at baseline

\begin{tabular}{|c|c|}
\hline Variable & $\begin{array}{l}\text { Mean } \pm \text { SEM (median; } \\
\text { range) or number }(\%)\end{array}$ \\
\hline \multicolumn{2}{|l|}{ Grading } \\
\hline G2 & $49(96.0 \%)$ \\
\hline G3 & $1(2.0 \%)$ \\
\hline Gx (no grading available) & $1(2.0 \%)$ \\
\hline \multicolumn{2}{|l|}{ Histology } \\
\hline Adenocarcinoma & $44(86.3 \%)$ \\
\hline Mucoid carcinoma & $7(13.7 \%)$ \\
\hline \multicolumn{2}{|l|}{ CS Mason score } \\
\hline CS II & $1(2.0 \%)$ \\
\hline CS III & $48(94.1 \%)$ \\
\hline Tumor not palpable & $2(3.9 \%)$ \\
\hline \multicolumn{2}{|l|}{ Preoperative UICC staging } \\
\hline cUICC II (uT3 uN0) & $32(62.7 \%)$ \\
\hline cUICC III (uT3 uN1) & $19(37.3 \%)$ \\
\hline \multicolumn{2}{|l|}{ ypTNM classification } \\
\hline урT0N0M0 & $4(7.8 \%)$ \\
\hline ypT1N0M0 & $5(9.8 \%)$ \\
\hline ypT1N1M0 & $1(2.0 \%)$ \\
\hline ypT2N0M0 & $13(25.5 \%)$ \\
\hline ypT3N0M0 & $16(31.4 \%)$ \\
\hline ypT3N1M0 & $9(17.6 \%)$ \\
\hline ypT3N2M0 & $3(5.9 \%)$ \\
\hline \multicolumn{2}{|l|}{ Dukes } \\
\hline Dukes A & $17(33.3 \%)$ \\
\hline Dukes B & $18(35.3 \%)$ \\
\hline Dukes C & $13(25.5 \%)$ \\
\hline No Dukes classification available & $3(5.9 \%)$ \\
\hline \multicolumn{2}{|l|}{ Lymphatic tumor propagation } \\
\hline Yes & $1(2.0 \%)$ \\
\hline No & $45(88.2 \%)$ \\
\hline No data available & $5(9.8 \%)$ \\
\hline \multicolumn{2}{|l|}{ Vessels infiltrated by cancer tissue } \\
\hline Yes & $5(9.8 \%)$ \\
\hline No & $41(80.4 \%)$ \\
\hline No data available & $5(9.8 \%)$ \\
\hline \multicolumn{2}{|l|}{ Tumor response } \\
\hline Responder (ypT0-ypT2) & $23(45.1 \%)$ \\
\hline Non-responders (ypT3) & $29(54.9 \%)$ \\
\hline
\end{tabular}

\section{Statistics Procedures}

Data analysis was performed with SPSS (version 11.5). Normal distribution was calculated by the Kolmogorov-Smirnov procedure. Depending on the results of this test, comparisons between the groups were made using the parametric t-test or non-parametric tests (Mann-Whitney, Kruskal-Wallis, $\mathrm{Chi}^{2}$, Fisher's exact test). Observed survival was estimated with Kaplan-Meier and log rank procedures. Statistical significance was defined as $\mathrm{p} \pm 0.05$.

\section{Results}

Two-thirds of our study population were males. The mean age was 59.0 years at the time of surgery. The Apache score was low (0-4 points) in most patients $(73.2 \%)$, and ASA II was dominant (76.5\%) (table 1). 
Table 3. Calculation and comparison of survival time (months) depending on different factors (Kaplan-Meier test)

\begin{tabular}{|c|c|c|c|}
\hline Variable & Mean + SE (95\%-CI) & Log rank & $\mathrm{p}$-value \\
\hline \multicolumn{4}{|l|}{ Age at surgery, years } \\
\hline $26.0-50.9$ & $107 \pm 27(53-161)$ & 3.19 & 0.20 \\
\hline $51.0-60.9$ & $130 \pm 11(108-153)$ & & \\
\hline $61.0-73.3$ & $144 \pm 11(121-167)$ & & \\
\hline \multicolumn{4}{|l|}{ Apache score, points } \\
\hline $0-4$ & $128 \pm 11(105-151)$ & 2.11 & 0.34 \\
\hline $5-9$ & $135 \pm 8(118-153)$ & & \\
\hline 10 & $139 \pm 18(102-176)$ & & \\
\hline \multicolumn{4}{|l|}{ ASA score } \\
\hline ASA I & $104 \pm 19(66-142)$ & 0.87 & 0.64 \\
\hline ASA II & $132 \pm 9(112-151)$ & & \\
\hline ASA III & $152 \pm 11(130-175)$ & & \\
\hline \multicolumn{4}{|l|}{ Histology } \\
\hline Adenocarcinoma & $139 \pm 8(122-157)$ & 2.47 & 0.11 \\
\hline Mucoid carcinoma & $86 \pm 14(57-115)$ & & \\
\hline \multicolumn{4}{|l|}{ Preoperative UICC staging } \\
\hline cUICC II (uT3 uN0) & $139 \pm 10(119-159)$ & 0.51 & 0.47 \\
\hline cUICC III (uT3 uN1) & $104 \pm 11(82-126)$ & & \\
\hline \multicolumn{4}{|l|}{ TNM classification } \\
\hline T1N0M0-T1N1M0 & $92 \pm 16(59-124)$ & 0.48 & 0.78 \\
\hline T2N0M0 & $117 \pm 9(98-136)$ & & \\
\hline T3N0M0-T3N2M0 & $129 \pm 12(105-152)$ & & \\
\hline \multicolumn{4}{|l|}{ Dukes (modified according to AJCC 1988) } \\
\hline Dukes A (TisN0M0, T1N0M0) & $124 \pm 8(107-141)$ & 2.37 & 0.30 \\
\hline Dukes B (T2-4N0M0) & $150 \pm 9(132-167)$ & & \\
\hline Dukes C (each T, N1-3, M0) & $102 \pm 8(86-119)$ & & \\
\hline \multicolumn{4}{|l|}{ Vessels infiltrated by cancer tissue } \\
\hline Yes & $78 \pm 18(43-114)$ & 1.94 & 0.16 \\
\hline No & $136 \pm 9(117-154)$ & & \\
\hline \multicolumn{4}{|l|}{ Surgery } \\
\hline Radical anterior rectal resection (ARR) & $113 \pm 9(96-131)$ & 0.75 & 0.68 \\
\hline Abdominoperineal rectal extirpation (APRE) & $127 \pm 13(100-153)$ & & \\
\hline Pull-through procedure + laparoscopic APRE & $144 \pm 19(106-182)$ & & \\
\hline \multicolumn{4}{|l|}{ Local recurrence in small pelvis, follow-up } \\
\hline Yes & $107 \pm 1(106-109)$ & 0.07 & 0.79 \\
\hline No & $132 \pm 9(114-151)$ & & \\
\hline \multicolumn{4}{|c|}{ Metachronous distant metastasis during follow-up period } \\
\hline Yes & $77 \pm 8(60-95)$ & & \\
\hline No & $154 \pm 9(135-172)$ & 19.23 & $<0.01$ \\
\hline \multicolumn{4}{|l|}{ Tumor response } \\
\hline Responder (ypT0-ypT2) & $116 \pm 8(100-132)$ & & \\
\hline Non-Responders (урТ3) & $129 \pm 12(105-152)$ & 0.41 & 0.52 \\
\hline
\end{tabular}

$\mathrm{SE}=$ Standard error $; \mathrm{CI}=$ confidence interval .
Most patients suffered from a rectal adenocarcinoma (86.3\%) with a stage 2 grading (96\%) and a Mason score II (94.1\%). One third of the patients showed an endosonographically confirmed N1 tumor prior to surgery. The TNM staging after surgery showed $7.8 \% \mathrm{~T} 0,11.8 \% \mathrm{~T} 1,25.5 \% \mathrm{~T} 2$, and $54.9 \% \mathrm{~T} 3$ tumors. One third of the tumors were Dukes A and B, one quarter was Dukes C. Lymphatic tumor propagation and vessel infiltration by tumor tissue was present in 2 and $9.8 \%$ of the cases, respectively. Most patients (54.9\%) showed a tumor response after NRC (table 2).

We found no statistical difference of the patients' age at the time of diagnosis $(p=0.428)$ or at the time of surgery $(p=0.40)$ between males and females. We could also not find a significant difference of the Apache score $(\mathrm{p}=0.84)$, the ASA score $(\mathrm{p}=0.22)$, grading $(\mathrm{p}=0.32)$, the CS Mason score $(\mathrm{p}=0.67)$, the cUICC staging $(\mathrm{p}=0.30)$, the pTNM classification $(\mathrm{p}=0.6217)$, the lymphatic propagation ( $p=0.67$ ), tumor infiltration into vessels $(p=0.18)$, or tumor response $(p=0.31)$ between males and females.

Only the occurrence of metachronous distant metastasis during the follow-up period had a significant influence on the patients' survival. The occurrence of distant metastasis reduces the survival time to less than $50 \%$ of patients without distant metastasis (table 3 ).

Kaplan-Meier analysis also showed that the ypT classification had no influence on the patients' survival in contrast to ypN classification. Patients with ypN0 had a mean survival time of 145 months while patients with ypN1 (83 months) or ypN2 (67 months) showed a remarkable decrease of the mean survival period (table 4 ). 
Table 4. Calculation and comparison of survival time (months) depending on ypT and ypN classification (Kaplan-Meier test)

\begin{tabular}{lrll}
\hline Variable & Mean \pm SE $(95 \%-C I)$ & Log rank & p-value \\
\hline ypT classification & & & \\
ypT0 (responders) & $115 \pm 15(84-146)$ & 0.55 & 0.90 \\
ypT1 (responders) & $92 \pm 16(59-124)$ & & \\
ypT2 (responders) & $117 \pm 9(98-136)$ & & \\
ypT3 (non-responders) & $129 \pm 12(105-152)$ & & \\
\hline ypN classification & $145 \pm 8(128-162)$ & 6.03 & \\
ypN0 & $83 \pm 14(54-111)$ & & \\
ypN1 & $67 \pm 27(14-120)$ & & \\
ypN2 & & \\
\hline SE $=$ Standard error; CI = confidence interval. & \\
\hline
\end{tabular}

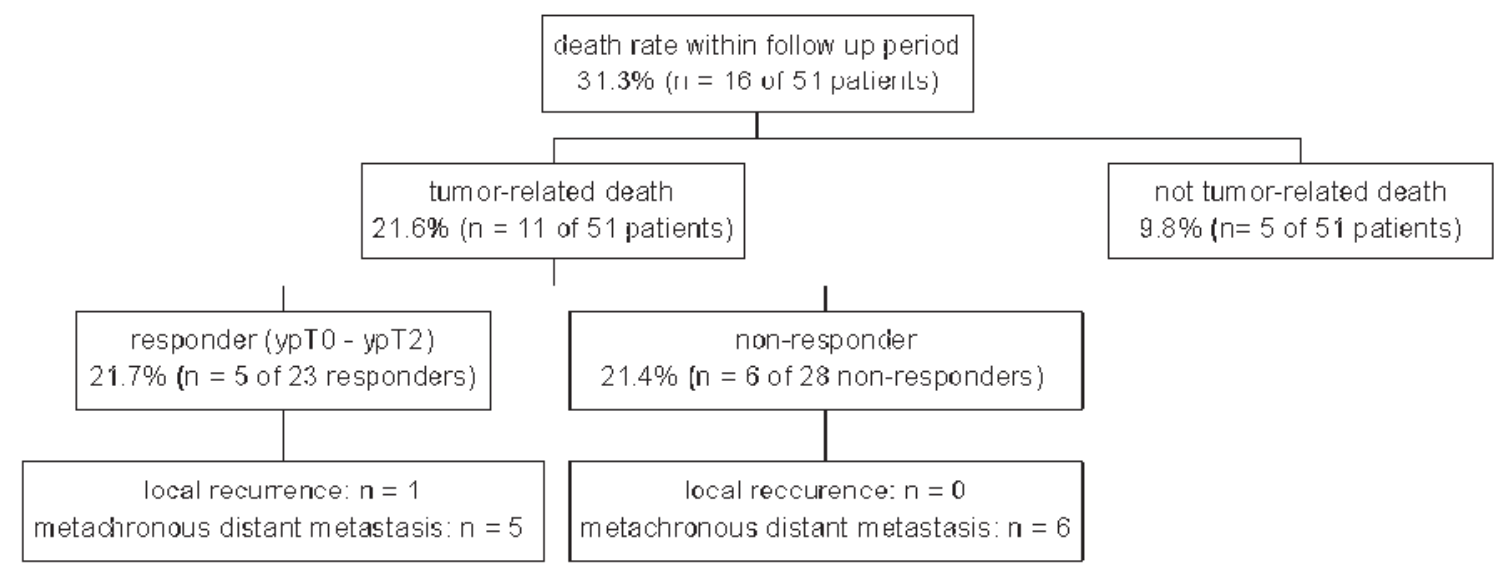

Fig. 1. Tumor-related death, local recurrence, and metachronous distant metastasis.
During a postoperative mean follow-up period of 8.2 years (range: 1 month to 14.5 years), 16 patients of the study population died (31.3\%). A fifth of all patients (21.6\%) died because of tumorrelated reasons. The tumor-related death rate among the 23 responders (ypT0-ypT2) during that period was $21.7 \%$, and the tumor-related death rate among the 28 non-responders (ypT3) was $21.4 \%$. All patients who died a tumor-related death showed metachronous distant metastasis but only one patient had local recurrence (fig. 1). The patients' cumulative survival rate after 5 years was $88 \%$ in non-responders and $86 \%$ in responders.

We were able to collect data about local recurrence, distant metastasis, and response rate in 19 responders and 25 non-responders. Among these patients, the rate of local recurrence during the follow-up period was $4 \%(n=1)$ in responders and $5.3 \%(n=1)$ in non-responders $(\mathrm{p}=0.68)$. The rate of metachronous distant metastasis was $31.6 \%(n=6)$ in responders and $32 \%(n=8)$ in nonresponders $(p=0.97)$. The total rate of metachronous distant metastasis in all patients investigated was 31.8\% (14/44 patients).

\section{Discussion}

This study investigated patients suffering from endosonographically confirmed T3 rectal cancer. The mean age of the patients at the time of surgery (59 years) is in accordance with other investigations [7-10]. A higher percentage rate of males with rectal cancers (male:female ratio 2:1) is also described in other trials [8-13]. Therefore, we conclude that our study population is comparable with the literature.

The German guidelines for the management of colorectal cancer were fundamentally changed in 2004 [2]. Neoadjuvant therapy was defined to be a new standard for lymph node-positive rectal cancers as well as for T3 rectal cancers. This change was partly based on the findings of the German Rectal Cancer Study Group [14]. They investigated 800 patients with rectal cancer treated with primary resection followed by an adjuvant therapy or with a neoadjuvant regime 4-6 weeks prior to surgery. In both groups the radiochemotherapeutical regime (50.4 Gy; 5-FU i.v.) was the same. Although the total survival rate showed no differences ( $74 \mathrm{vs.} 76 \%$ ), the rate of local recurrence was twice as high in the adjuvant section of the trial in comparison to the neoadjuvant regime ( 13 vs. $6 \%$ ).

The aim of a neoadjuvant treatment prior to surgery is to increase the possibility of an $\mathrm{R} 0$ resection of the rectal cancer. This success is assessed by the induction of a complete tumor regression (ypT0) or by analyzing the extent of cancers downgraded by neoadjuvant therapy (i.e. from T3 to T2 or from T3 to T1). According to Willeke et al. [15], a complete tumor regression is an important surrogate marker for the assessment of neoadjuvant treatment. Based on the findings of Rödel et al. [16], a complete tumor regression after neoadjuvant pretreatment leads to an improvement of the 5-year disease-free survival rate in comparison to patients not responding to this pretreatment (86 vs. $63 \%$ ). In contrast, Pucciar- 
elli et al. [8] could not confirm those findings; thus, it still seems to be unclear whether tumor response is an important factor regarding patient survival or not.

For this reason, it is necessary to investigate the influence of several factors (age, TNM, histology, Dukes, vessel infiltration, surgical procedure, lymphatic tumor propagation, and response rate) on patients treated for rectal cancer. In order to have a homogeneous cohort we only included patients with endosonographically confirmed T3 rectal cancer, curative elective surgery, R0 resection, and a metastatic-free disease prior to surgery. The accuracy of endosonography for T staging of rectal cancer ranges from 80 to $95 \%$ compared with computed tomography (65-75\%) and magnetic resonance imaging (MRI) (75-85\%) [17]; however, its accuracy for the assessment of rectal wall penetration (67-93\%) and determination of the nodal status $(64-88 \%)$ is lower $[18,19]$. This might have influenced some findings in our trial because the decision to classify the tumor response of the patients was made by using parameters (i.e. histology) evaluated during surgery and by comparing them with preoperative endosonographic findings. The impact of this influence is unknown but Daniels et al. [20] reported a failure rate of $18 \%$ for $\mathrm{T} 3$ and $\mathrm{T} 4$ staging in one group in the trial of the German Rectal Cancer Study Group.

It is difficult to compare the response rates of neoadjuvant pretreated rectal cancer patients with our results due to many studies using heterogeneous cohorts consisting of T3 and T4Nx tumors [21-26]. Some authors employed the UICC system for the calculation of the response rate [14]. Trials published by Rich et al. [27] investigated patients with T3 rectal cancer, and Rödel et al. [28] investigated those with T4 rectal cancer. For this reason, the percentage rate of ypT0 rectal cancers after neoadjuvant therapy using 5 -FU intravenously ranges from 6 to $33 \%$. The response rate of our study amounts to $7.8 \%$ (only the patients with ypT0N0M0 tumors) or $45.1 \%$ (including all cases up to ypT2N0M0 with and without positive lymph nodes). Tulchinsky et al. [9] reported 97 rectal cancer patients with pretreatment staging from uT2N0 to uT4N0. They also found a small rate of uTON0 tumors after chemoradiation with 50.4 Gy and 5-FU (4/97 cases; $4.1 \%)$ prior to surgery although a downstaging was achieved in $45 \%$ of the patients. Focusing on the rate of ypT0N0M0 tumor response alone and comparing this with the literature, our neoadjuvant regime (5-FU + folinic acid; $50.4 \mathrm{~Gy}$ ) might not produce a response rate superior to that resulting after continuous venous 5 -FU infusion alone but is able to reduce the T3 tumor size remarkably prior to surgery.

Our results show that the survival time of patients with rectal cancer treated with neoadjuvant therapy was not significantly influenced by age, Apache and ASA score, histology of the tumor, preoperative UICC staging, TNM classification, Dukes classification, cancer infiltration of vessels, type of surgery, local recurrence, and tumor response (table 3).

The rate of local recurrence in our trial could only be calculated based on a very small number of cases. Only two local recurrences occurred (one in each group of responders and non-responders); however, the total number ( $4.5 \%$ or $2 / 44$ patients) is too small to draw valid conclusions. Additionally, this rate has to be compared with other studies that also used NRC to treat patients with T3 rectal cancer. Brown et al. [11] reported a rate of local recurrence of $4.7 \%(1 / 21)$ in patients with and of $10.3 \%(7 / 68)$ in patients without complete pathologic response suffering from TNM stage II or III rectal cancer. The German Rectal Cancer Trial also showed a low local recurrence rate (6\%) [7].

Although we found no higher rate of distant metastasis in responders and non-responders, we were able to demonstrate that the occurrence of metachronous distant metastasis nearly reduces the patients' survival time after surgery to $50 \%$ in comparison to patients without distant metastasis. This is comparable to the results of Chan et al. [29], who found a 5-year relapse-free survival rate of $42 \%(\mathrm{~N} 1-\mathrm{N} 2)$ compared with $85 \%$ (N0) in patients after NRC prior to surgery.

Although we could not find significant differences in local recurrence, distant metastasis, and ypN0/ypN1-2 classified survival between responders and non-responders, this does not mean that tumor response has no influence at all. The main reason for this problem is that the amount of patients being investigated at the time of publication might be too small for obtaining significant results (i.e. local recurrence). Thus, it is necessary to investigate larger cohorts of T3 rectal cancer patients in future studies.

In our historical cohort analysis, we describe the multimodal treatment years before implementation in the German guidelines. Therefore, on the one hand, we find it interesting to analyze this 'study group' and to compare the data with the published literature. On the other hand, we are not able to correlate our data with modern aspects of standard diagnostics (pelvic MRI, MERCURY criteria, $\mathrm{CRM} \pm$, etc.). As a result, the additional information is restricted but of historical interest.

\section{Conclusion}

Our results illustrate the effectiveness of an NRC (50.4 Gy; 5-FU plus folinic acid) in patients with T3 rectal cancer. Most patients showed tumor response although the rate of ypT0N0M0 response was $7.8 \%$. We found no significant difference of tumor survival between responders and non-responders apart from the occurrence of distant metastasis, and patients with $y p N 1 / \mathrm{N} 2$ face a significantly shorter time of tumor survival. It is necessary to investigate larger cohorts of patients in order to obtain more data for valid conclusions in the future.

\section{Disclosure Statement}

The authors declare that they have no conflict of interests. The authors receive no funding. The data and manuscript are generated in accordance with the Declaration of Helsinki.

Author contributions: AT and HGH participated in the design of the study and drafted the manuscript. MD performed the statistical analysis and wrote the manuscript. DA helped to draft the manuscript. HRR conceived of the study, participated in its design, and helped to draft the manuscript. All authors read and approved the final manuscript. 


\section{References}

1 Krebsregister Rheinland-Pfalz: Krebs in RheinlandPfalz. Inzidenz und Mortalität im Jahr 2000. Mainz Leipziger Messe, 2000. www.krebsregister-rheinlandpfalz.de.

2 Schmiegel W, Pox C, Adler G, et al: S3 guidelines colorectal cancer. Z Gastroenterol 2004;42:1129-1177.

3 Kerr J, Engel J, Eckel R, Holzel D: Survival for recta cancer patients and international comparisons. Ann Oncol 2005; 16:664-672

4 Junginger T, Lorenz W: Diagnostik, Therapie und Nachsorge des kolorektalen Karzinoms. Interdisziplinäre S3-Leitlinie. www.onkodin.de/zms/content/e2/ e22972/e23955/e24993/index_ger.html (last modification: April 8, 2004) (accessed June 16, 2007).

5 Balch GC, De Meo A, Guillem JG: Modern management of rectal cancer: a 2006 update. World J Gastroenterol 2006;12:3186-3195.

6 Sauer R: Adjuvant vs. neoadjuvant combined modality treatment for locally advanced cancer: first results of the German Rectal Cancer Study (CAO/ARO/AIO 94). Int J Radiat Oncol Biol Phys 2003;57:124-125.

7 Klas JV, Rothenberger DA, Wong WD, Madoff RD: Malignant tumors of the anal canal. The spectrum of disease, treatment and outcome. Cancer 1996;85:16861693.

8 Pucciarelli S, Toppan P, Friso ML, et al: Complete pathologic response following preoperative chemoradiation therapy for middle and lower rectal cancer is not a prognostic factor for a better outcome. Dis Colon Rectum 2004:47:1798-1807.

-9 Tulchinsky H, Rabau M, Shacham-Shemueli E, et al: Can rectal cancer with pathologic $\mathrm{T} 0$ after neoadjuvant chemoradiation (ypT0) be treated by transanal excision alone? Ann Surg Oncol 2006;13:347-352.

10 Willeke F, Horisberger K, Kraus-Tiefenbacher U, et al: A phase II study of capecitabine and irinotecan in combination with concurrent pelvic radiotherapy $(\mathrm{Ca}-$ pIri-RT) as neoadjuvant treatment of locally advanced rectal cancer. Br J Cancer 2007;96:912-917.
Brown CL, Ternent CA, Thorson AG, et al: Response to preoperative chemoradiation in stage II and II rectal cancer. Dis Colon Rectum 2003;46:1189-1193.

12 Bujko K, Nowacki MP, Nasierowska-Guttmejer A, et al: Sphincter preservation following preoperative radiotherapy for rectal cancer: report of a randomised trial comparing short-term radiotherapy vs. conventionally fractionated radiochemotherapy. Radiother Oncol 2004; 72:15-24.

3 Coco C, Valentini V, Manno A, et al: Functional results after radiochemotherapy and total mesorectal excision for rectal cancer. Int J Colorectal Dis 2007;22: 903-910.

14 Sauer R, Becker H, Hohenberger W, et al: Preoperative versus postoperative chemoradiotherapy for rectal cancer. N Engl J Med 2004;351:1731-1740.

15 Willeke F, Horisberger K, Post S: Neoadjuvante Therapie beim Rektumkarzinom. Auf dem Weg zur individuellen Therapie. Z Gastroenterol 2006;44:1053-1063.

16 Rödel C, Martus P, Papadoupoulos T, et al: Prognostic significance of tumor regression after preoperative chemoradiation therapy for middle to lower rectal cancer. J Clin Oncol 2005;23:8688-8698.

17 Siddiqui AA, Fayiga Y, Huerta S: The role of endoscopic ultrasound in the evaluation of rectal cancer. Int Semin Surg Oncol 2006;3:36-43

18 Pijl ME, Chaoui AS, Wahl RL, van Oostayen JA: Radiology of colorectal cancer. Eur J Cancer 2002;38:887898.

19 Schaffzin DM, Wong WD: Endorectal ultrasound in the preoperative evaluation of rectal cancer. Clin Colorectal Cancer 2004;4:124-132.

20 Daniels IR, Fisher SE, Brown, G, Heald RJ, Moran RJ: Accurate staging, selective preoperative therapy and optimal surgery improves outcome in rectal cancer: a review of the recent evidence. Colorectal Dis 2007;9: 290-301.
1 Luna-Perez P, Rodriguez-Ramirez S, Rodriguez-Coria $\mathrm{D}$, et al: Preoperative chemoradiation therapy and anal sphincter preservation with locally advanced rectal adenocarcinoma. World J Surg 2001;25:1006-1011.

22 Metha VK, Poen J, Ford J, et al: Radiotherapy, concomitant protracted-venous-infusion 5-fluorouracil and surgery for ultrasound-staged T3 or T4 rectal cancer. Dis Colon Rectum 2001;44:52-58.

23 Movsas B, Hanlon AL, Lanciano R, et al: Phase 1 dose escalating trial of hyperfractionated pre-operative chemoradiation for locally advanced rectal cancer. Int J Radiat Oncol Biol Phys 1988;42:43-50.

24 Rau B, Hohenberger P, Gellermann J, et al: T4 rectal carcinoma. Surgical and multimodal therapy. Chirurg 2002;73:147-153.

25 Rullier E, Goffre B, Bonnel C, Zerbib F, Caudry M, Saric J: Preoperative radiochemotherapy and sphincter-saving resection for T3 carcinomas of the lower third of the rectum. Ann Surg 2001;234:633-640.

26 Tjandra JJ, Reading DM, McLachlan SA, et al: Phase II clinical trial of preoperative combined chemoradiation for T3 and T4 resectable rectal cancer: preliminary results. Dis Colon Rectum 2001;44:1113-1122.

27 Rich TA, Skibber JM, Ajani JA, et al: Preoperative infusional chemoradiation therapy for stage T3 rectal cancer. Int J Radiat Oncol Biol Phys 1995;32:1025-1029.

28 Rödel C, Grabenbauer GG, Matzel KE, et al: Extensive surgery after high-dose preoperative chemoradiotherapy for locally advanced recurrent rectal cancer. Dis Colon Rectum 2000;43:312-319.

29 Chan CL, Bokey EL, Chapuis PH, Renwick AA, Dent OF: Local recurrence after curative resection for rectal cancer is associated with anterior position of the tumour. Br J Surg 2006;93:105-112. 